\title{
Periodic Wall Blow/Suction Perturbation Evolution in Turbulent Boundary Layer*
}

\author{
Gangli Hao $^{1,2}$, Nan Jiang ${ }^{1,3 \#}$ \\ ${ }^{1}$ Department of Mechanics, School of Mechanical Engineering, Tianjin University, Tianjin, China, \\ ${ }^{2}$ Shijiazhuang University of Economics, School of Engineering, Shijiazhuang, China, \\ ${ }^{3}$ Tianjin Key Laboratory of Modern Engineering Mechanics, Tianjin, China, \\ Email: hg1920@126.com, ${ }^{*}$ nanj@tju.edu.cn
}

Received July 12, 2012; revised August 12, 2012; accepted August 19, 2012

\begin{abstract}
Time sequence signals of instantaneous longitudinal and normal velocity components at different longitudinal and normal positions in a turbulent boundary layer have been finely measured simultaneously by IFA 300 constant temperature anemometer and double-sensor hot-wire probe with sampling resolution higher than the frequency that corresponds to the smallest time scale of Kolmogorov dissipation scale before/after introducing artificial periodic blow/suction perturbation. The period-phase-average technique is applied to extract the periodic waveforms of artificial perturbation from instantaneous time sequence signals of longitudinal and normal turbulence background. Experimental investigation is carried out on the attenuation characteristics of periodic perturbation wave with different frequency along longitudinal direction and normal direction in a turbulent boundary layer. The amplitude distributions of longitudinal and normal disturbing velocity component for different perturbation frequencies are measured at different downstream and normal positions in turbulent boundary layer. The amplitude growth rate of artificial periodic perturbation wave is calculated according to flow instability theory. The experimental results are compared and in consistent with the theoretical and numerical results.
\end{abstract}

Keywords: Artificial Periodic Perturbation; Blow/Suction; Turbulent Boundary Layer; Hot-Wire Anemometry

\section{Introduction}

Since Kline (1967) [1] discovered the coherent largescale low-speed streak structure for turbulence production in the near wall region of turbulent boundary layer, Whether the evolution of burst is related to near wall flow conditions or outer flow conditions is not given a clear conclusion. At the beginning, it was generally considered streak structures and burst processes are the feature phenomena in the near wall region influenced only by wall conditions directly, and it is independent of flow conditions in the outer region. Blackwelder (1972) [2] discovered that the movements in the viscous layer are strongly correlated with that in the outer region. Offen et al. [3] (1974)'s experiments also indicated that the burst is deeply influenced by the outer flow. This is reasonable to explain the existence of large-scale vortex in the turbulent boundary layer and the energy source for turbulence generated by bursts. Rao [4] and Kim [5] normal-

\footnotetext{
"This work was jointly supported by the National Natural Science Foundation of China (Grant Nos. 10832001 and 11272233), National key basic research and development program (plan 973) (NO. 2012CB720101). This work was jointly supported by Hall of Hebei province science and technology of China (11213947).

${ }^{\#}$ Corresponding author.
}

ized the average burst cycle by using boundary layer's external parameters (boundary layer thickness $\delta$ and free flow velocity $U_{\infty}$ ) and found that the normalized average burst cycle is independent of the Reynolds number. Laufer [6] concluded that $\bar{T} U_{\infty} / \delta \approx 5$ by $T^{+}=\bar{T} u_{\tau}^{2} / v=0.65 \operatorname{Re}_{\theta}^{0.75}$ in the range of $500<\operatorname{Re}_{\theta}<10^{4}$ and corroborated his experimental results. This discovery led the researches to emphasize on the relationship between inner boundary layer and outer boundary layer. The experimental results of Rao [4], Kim [5] and Offten [3] showed that the generation of burst is related to the outer flow of boundary layer, which can explain reasonably the existence of large-scale vortex in the turbulent boundary layer and the energy source of turbulence production from burst, but the researches of Chamber [7], Willmarth [8], Luchick [9], and Shu [10] are more inclined to the inner parameter's impact on the coherent structure burst, they believed that the impact of wall shear is more direct and important. The debate on whether the burst temporal scale is impacted by outer or inner flow conditions is essentially on the generation and maintenance mechanism of burst. The occurrence and evolution of burst plays a key role in the generation and 
maintenance of turbulence and the transportation of turbulence energy, thereby studies on the flow conditions influencing the generation and evolution of burst is of great significance.

Inspired by the success of imposing artificial periodic perturbation in lamina boundary layer transition experiment, adopting different methods to introduce artificial periodic perturbation to turbulent boundary layer and examining the influence of artificial periodic perturbations on the burst can solve, from another point of view, the problem of whether the burst time scale is influenced by outer or inner flow conditions. Hussain et al. $[11,12]$ introduced perturbation in the fully developed two-dimensional turbulent channel flow by using electromagnetic excitation method and got a result which is in agreement well with Landahl's [13] linear theory. V. V. Kozlov, et al. [14] studied the developing process of perturbing wave in Poiseuille flow. Yao [15] introduced organized artificial perturbation in water channel experiments by mechanical vibrating ribbon, studied preliminarily the development and evolution of low-frequency perturbation wave along the longitudinal direction and the influence of perturbation in outer region on bursts in inner layer.

Theoretical researches are mainly focused on the development of sinusoidal wave perturbation by the method of lamina instability. Researches in this field started from M. Landahl's waveguide model. The numerical results are in agreement with Willmarth's [16] experimental results.

\section{Experiment Technology and Apparatus}

In this experiment, periodic artificial blow/suction perturbations of different frequencies are introduced into a turbulent boundary layer through a spanwise slot by exciting a woofer vibrating under the wall in order to manipulate the multi-scale flow structure in turbulent bound- ary layer. The evolution and the influence of periodic blow/suction perturbation on the turbulent boundary layer are investigated experimentally. Periodic blowing and suction disturbance provides a simple method for wall-bounded turbulence control because the frequency and amplitude of blowing \& suction disturbance is able to be quantified and adjusted easily and accurately.

Time sequence signals of longitudinal and normal velocity components at different longitudinal and normal positions in a turbulent boundary layer have been finely measured before/after introducing periodic blow/suction perturbation by IFA300 constant temperature anemometer and TSI-1243 double-sensor hot-wire probe with sampling resolution higher than the frequency that corresponds to the smallest time scale of turbulence. In this paper, experimental results under the conditions of different perturbation were compared and analyzed statistically. Sampling frequency of the experiment is $20 \mathrm{KHz}$; measuring time for each sampling space point is 13.1072 seconds, and data volume is 262,144 .

The experiment is carried out in the low-turbulent level wind tunnel of the Fluid Mechanics Laboratory of Tianjian University. The test section of wind tunnel is made of wood; the cross-section of the test section is a rectangular without corner, with $4500 \mathrm{~mm}$ in length, 450 $\mathrm{mm}$ in height, and $350 \mathrm{~mm}$ in width. Wind velocity in the test section can be continuously adjusted from $1.0 \mathrm{~m} / \mathrm{s}$ to $40.0 \mathrm{~m} / \mathrm{s}$, and the background turbulent level is less than $0.07 \%$. Experimental apparatus arrangement is shown in Figure 1: the experimental flat-plate is $2000 \mathrm{~mm}$ in length, $350 \mathrm{~mm}$ in width and $2 \mathrm{~mm}$ in thickness; the leading edge of the flat-plate, facing straightly the coming flow, is wedge-shaped; local perturbation is introduced through a spanwise slim slit of $5 \mathrm{~mm}$ longitudinal wide and $345 \mathrm{~mm}$ spanwise long on the smooth flat plate; the slim slit is located $1000 \mathrm{~mm}$ downstream away from

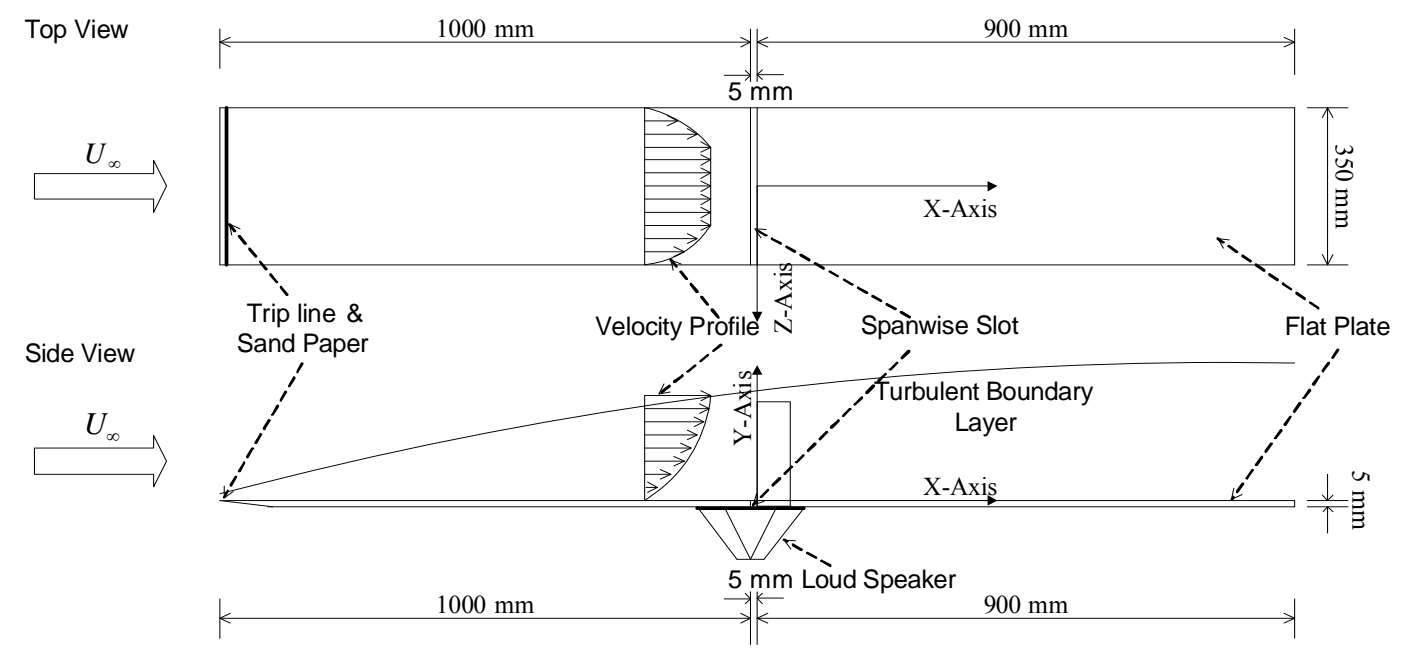

Figure 1. Experimental apparatus for suction and blowing (unit: mm). 
the leading edge of the flat plate, The origin of the coordinate axes is located at the center point of the downstream edge of the spanwise slot. The longitudinal direction is $x$ and the normal direction is $y$. A signal generator is used to produce sinusoidal wave signals with controllable frequency. The sinusoidal wave signals are amplified by a power amplifier to power a $300 \mathrm{~mm}$-diametered woofer periodically, introducing blow/suction perturbation airflow into the turbulent boundary layer, the amplitude of the perturbation is controlled by the amplifier's power output.

TSI Company's IFA300 constant temperature anemometer is used, together with CCTS-1193E automatic controlled three-dimensional coordinate frame and Thermalpro software package to carry out the instantaneous velocity measurement automatically. The sensor is a TSI1243-T1.5 $\times$ type hot-wire probe with double slantwise hot-wires made of $5 \mu \mathrm{m}$ diameter tungsten fine filament, and the probe is calibrated on the TSI-1128 hot-wire probe calibrator before used.

The mean velocity profiles with/without different frequency perturbations at two locations $X=5 \mathrm{~mm} X=5$ $\mathrm{mm} X=10 \mathrm{~mm} X=20 \mathrm{~mm} X=30 \mathrm{~mm}$ downstream respectively the disturbing source are plotted by inner variable unit in Figure 2 where $u^{+}=u / u_{*}$ and $y^{+}=y u_{*} / v$ are the normalized streamwise mean velocity and the distance from the wall by the skin friction velocity $u_{*}$ and the kinematics' viscosity $v$. The undisturbed longitudinal mean velocity profile fits to the Spalding law well. As for the disturbed profiles, lower mean velocities are found in the buffer layer where the logarithmic region is not affected. As the flow develops downstream, this phenomena gradually disappears and the disturbed velocity profiles recover to the undisturbed state at $X=30 \mathrm{~mm}$. This implies that the effect domain of blowing/suction periodic disturbance is limited and the blowing/suction periodic disturbance cannot be propagated downstream far away in turbulent boundary layer. The blowing/suction periodic disturbance is fused by the receptivity of turbulent boundary layer and decays gradually.

\section{Experimental Results and Analysis}

\subsection{Experimental Data Analysis Methods}

The eigenwaveform of the periodic artificial perturbation wave is educed by the phase-align period average technique. It is convenient to represent the velocity field as a superposition of three parts:

$$
u_{j}=\bar{u}_{j}+\tilde{u}_{j}+u_{j}^{\prime}
$$

Here $j=1,2,3$ stands for velocity components of three directions, $\bar{u}_{j}$ is the mean velocity over a long time period, $\tilde{u}_{j}$ is phase average of periodic perturbation,

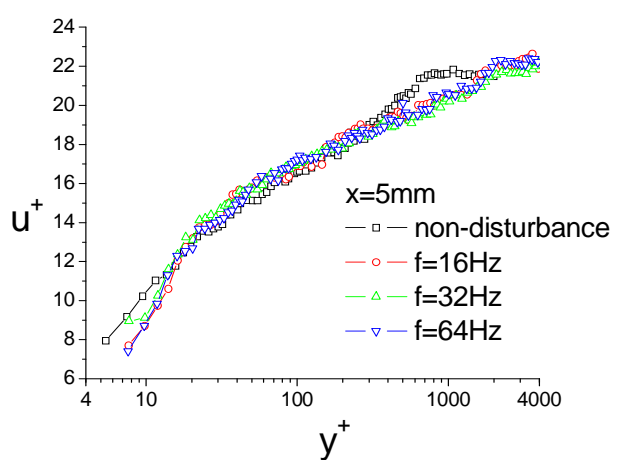

(a)

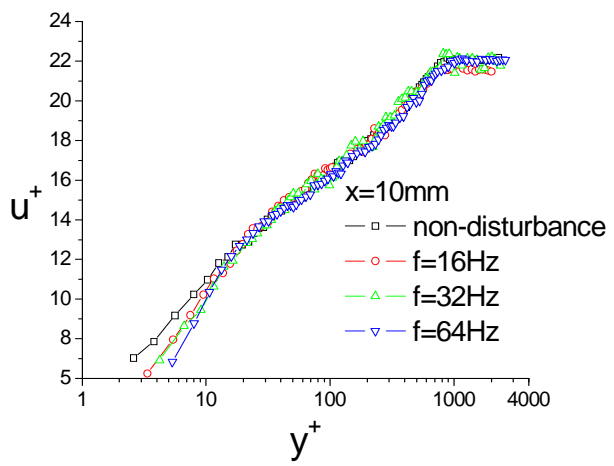

(b)

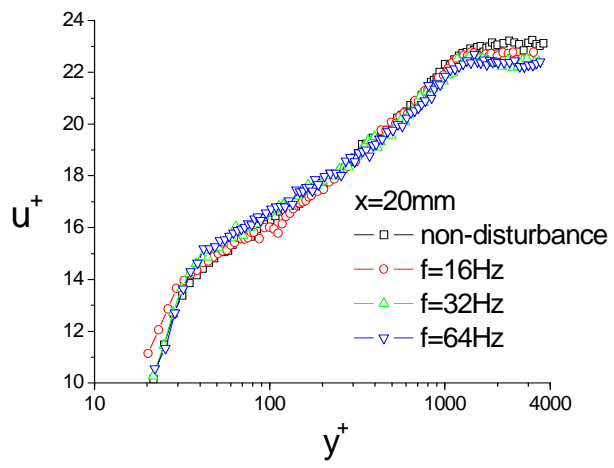

(c)

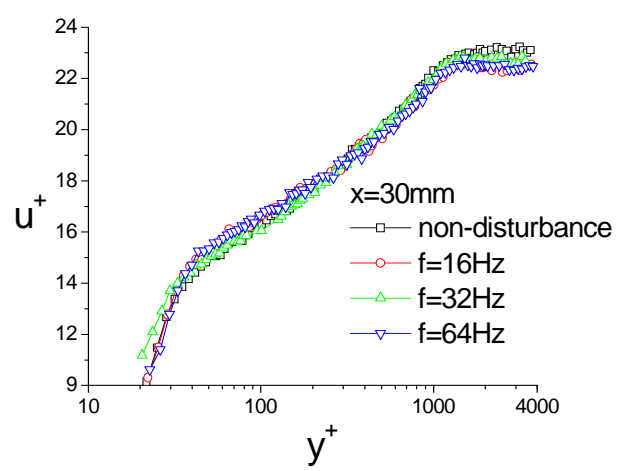

(d)

Figure 2. Mean velocity profile of turbulent boundary layer downstream suction and blowing perturbation ((a) disturbance source downstream $5 \mathrm{~mm}$; (b) disturbance source downstream $10 \mathrm{~mm}$; (c) disturbance source downstream 20 mm; (d) disturbance source downstream $30 \mathrm{~mm}$ ). 
and $u_{j}^{\prime}$ corresponds to the background random fluctuation. The mean velocity $\bar{u}_{j}$ can be determined by average over a long time (long time average of $\tilde{u}_{j}$ and $u_{j}^{\prime}$ are $\left.\overline{u_{j}^{\prime}}=0, \bar{u}_{j}=0\right), \quad \tilde{u}_{j}$ can be obtained through the phase-align period average method, and the phase average of the instantaneous velocity then gives the sum of the mean and the organized wave. Denoting the phase average by \langle\rangle , we have

$$
\left\langle u_{j}\right\rangle=\bar{u}_{j}+\tilde{u}_{j}
$$

and then,

$$
\tilde{u}_{j}=\left\langle u_{j}\right\rangle-\bar{u}_{j}=\left\langle u_{j}-\bar{u}_{j}\right\rangle
$$

The instantaneous velocity is measured at seven longitudinal positions downstream from the perturbation source; the amplitude and eigenwaveform of the perturbation are measured by the phase-average technique.

It is usual to represent the two-dimensional perturbation wave as:

$$
\tilde{u}_{j}(x, y, t)=\frac{1}{2}\left\{\hat{u}(y) e^{i \alpha(x-c t)}+\hat{u}^{*}(y) e^{-i \alpha^{*}\left(x-c^{*} t\right)}\right\}
$$

where * stands for complex conjugate, $\hat{u}(y)$ is the twodimensional complex eigenmode, $\alpha=\alpha_{r}+i \alpha_{i}$ is the complex wavenumber, $\alpha_{r}$ being the longitudinal wave number and $\alpha_{i}$ the growth rate factor, and $c=c_{r}+i c_{i}$ is the complex wave speed.

The perturbation velocity amplitudes at two different longitudinal stations $x_{1}, x_{2}$ with the same distance $y$ from the wall

$$
\begin{aligned}
& \left|\tilde{u}\left(x_{1}, y, t\right)\right|=\left|\tilde{u}(y) e^{i\left(\alpha_{r} x_{1}-\omega t\right)} e^{-\alpha_{i} x_{1}}\right|=|\hat{u}(y)| e^{-\alpha_{i} x_{1}} \\
& \left|\tilde{u}\left(x_{2}, y, t\right)\right|=|\hat{u}(y)| e^{-\alpha_{i} x_{2}}
\end{aligned}
$$

The amplitudes ratio:

$$
\left|\tilde{u}\left(x_{2}, y, t\right)\right| /\left|\tilde{u}\left(x_{1}, y, t\right)\right|=e^{-\alpha_{i}\left(x_{2}-x_{1}\right)}
$$

that is

$$
\left|\tilde{u}\left(x_{1}, y, t\right)\right| /\left|\tilde{u}\left(x_{2}, y, t\right)\right|=e^{\alpha_{i}\left(x_{2}-x_{1}\right)}
$$

there by,

$$
\alpha_{i}=\frac{\ln \left(\left|\tilde{u}\left(x_{1}, y, t\right)\right| /\left|\tilde{u}\left(x_{2}, y, t\right)\right|\right)}{x_{2}-x_{1}}
$$

If the perturbation amplitude increases along the longitudinal direction, thus

$$
\left|\tilde{u}\left(x_{2}, y, t\right)\right| /\left|\tilde{u}\left(x_{1}, y, t\right)\right|=e^{-\alpha_{i}\left(x_{2}-x_{1}\right)}>1
$$

that is

$$
\left|\tilde{u}\left(x_{1}, y, t\right)\right| /\left|\tilde{u}\left(x_{2}, y, t\right)\right|=e^{\alpha_{i}\left(x_{2}-x_{1}\right)}<1,
$$

and then $\alpha_{i}<0$ is obtained according to $x_{2}-x_{1}>0$.
If perturbation attenuations along the longitudinal direction, thus

$$
\left|\tilde{u}\left(x_{2}, y, t\right)\right| /\left|\tilde{u}\left(x_{1}, y, t\right)\right|=e^{-\alpha_{i}\left(x_{2}-x_{1}\right)}<1
$$

that is

$$
\left|\tilde{u}\left(x_{1}, y, t\right)\right| /\left|\tilde{u}\left(x_{2}, y, t\right)\right|=e^{\alpha_{i}\left(x_{2}-x_{1}\right)}>1,
$$

and then $\alpha_{i}>0$ is obtained according to $x_{2}-x_{1}>0$.

It is known from above that for the same $y$ value, wave amplitudes at different longitudinal stations of $x$, perturbation wave amplitude growth rate $\alpha_{i}$ can be calculated from Equation (7).

\subsection{Perturbation Wave Amplitude Distribution along $x-y$ Plane}

Figure 3 shows the period-averaged waveforms of longitudinal velocity component at five different vertical locations $10 \mathrm{~mm}$ downstream the blow/suction disturbance source with different frequencies. It is can be seen that the period-averaged waveforms are typical sinusoidal disturbance.

Figure 4 are the perturbation wave amplitude distributions of $16 \mathrm{~Hz}, 32 \mathrm{~Hz}, 64 \mathrm{~Hz}$ across the turbulent boundary layer at different longitudinal stations downstream the disturbance source. The perturbation wave amplitude response in the outer region $\left(y^{+}>100\right)$ caused by the 16 $\mathrm{Hz}$ and $32 \mathrm{~Hz}$ perturbation is much larger than that in the near wall region $\left(y^{+}<100\right)$. It implies that the periodic perturbation in the near wall region causes the response on the outer region, and it shows further the inner and outer boundary layer are not absolutely separated and they are sensible closely each other. The emergence of inner layer's burst is intimately related to the outer boundary layer's flow conditions.

While the $64 \mathrm{~Hz}$ perturbation distorts seriously in the turbulent boundary layer, the attenuations are relatively rapid, and fails to produce relatively large amplitude perturbation wave's response in the outer region $\left(y^{+}>\right.$ $100)$, which is related to the inherent predominant eigenmode of the turbulent boundary layer, as shown in Figure 5. Figures 5(a)-(d) show, in different longitudinal stations and the same normal position $y^{+}=12$, before and after different frequencies perturbations different frequencies are introduced, the velocity fluctuation power spectrum across the scale parameters (frequency components). It is found that there exists a most energetic scale $\mathrm{a}=6$ (corresponding to $128 \mathrm{~Hz}$ ) when no perturbation is introduced. After $16 \mathrm{~Hz}$ perturbation has been introduced, the turbulent energy decreases on the Scales $1 \sim 7$ and increases on Scales $8 \sim 11$, the peak change to Scale 9 (corresponding to $16 \mathrm{~Hz}$ ). Similarly, for the perturbation frequency of $32 \mathrm{~Hz}$ and $64 \mathrm{~Hz}$, the largest containing energy scale changes respectively to $\mathrm{a}=8(32 \mathrm{~Hz})$ and 


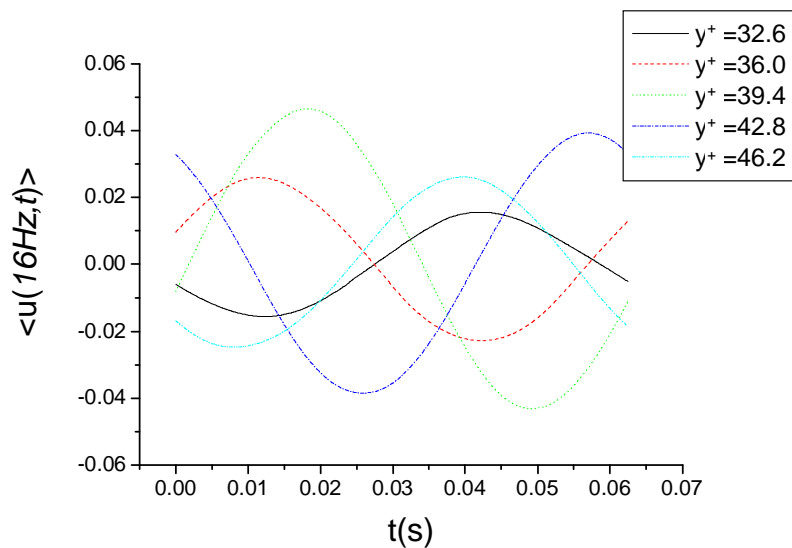

(a)

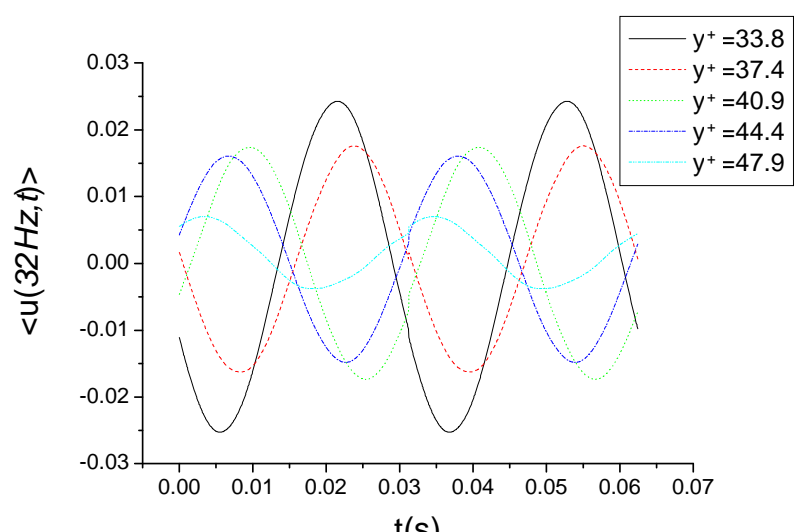

(b)

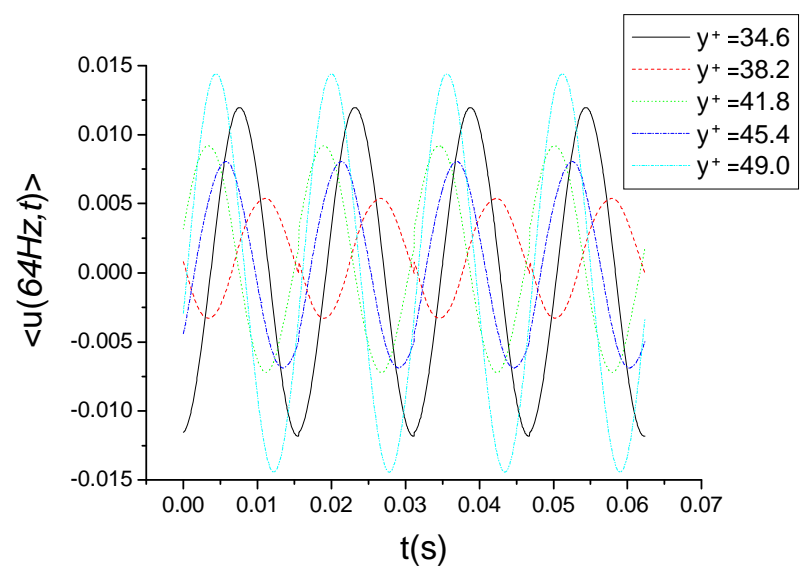

(c)

Figure 3. Phase average of periodic suction and blowing velocity components at different locations from the wall downstream the wave-maker $10 \mathrm{~mm}$ ((a) $16 \mathrm{~Hz}$, (b) $32 \mathrm{~Hz}$, (c) $64 \mathrm{~Hz}$ ).

$a=7(64 \mathrm{~Hz})$. This shows that different frequencies periodic perturbations change the inherent frequency components of the velocity fluctuation in turbulent boundary layer. $64 \mathrm{~Hz}$ perturbation is the nearest frequency to the inherent main frequency of the velocity fluctuation in turbulent boundary layer, and the interaction with the

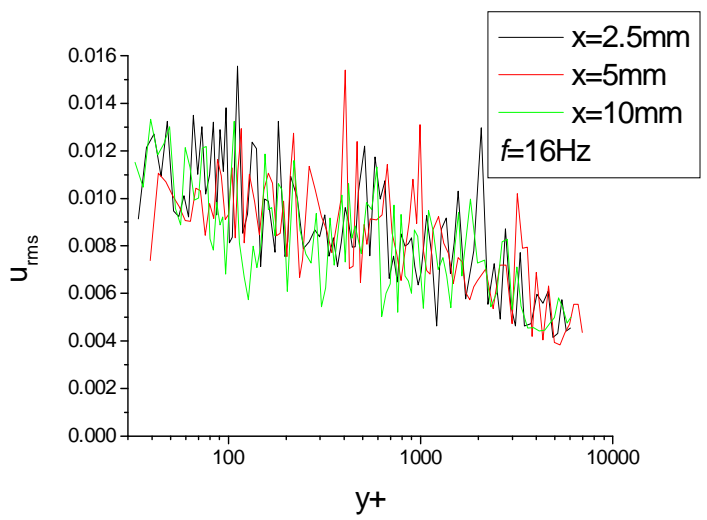

(a)

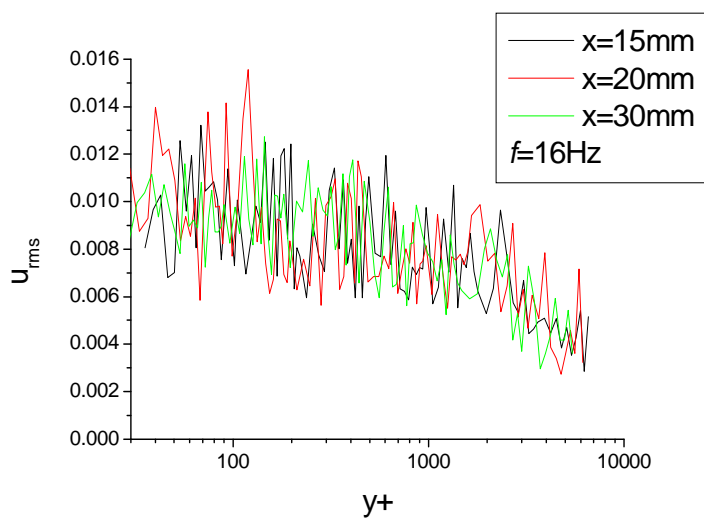

(b)

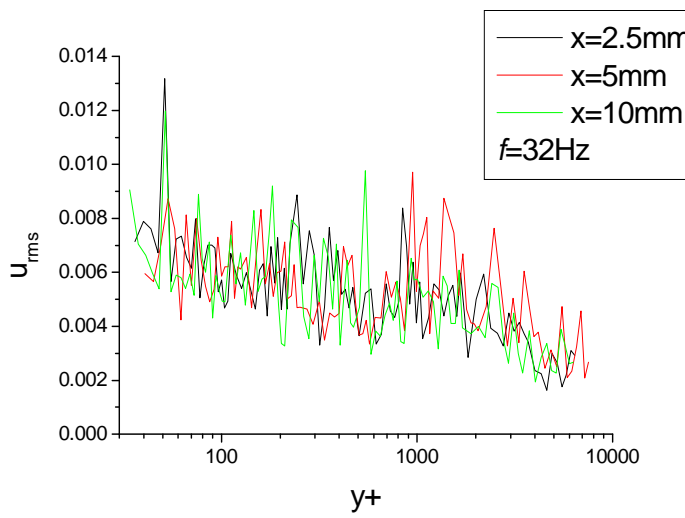

(c)

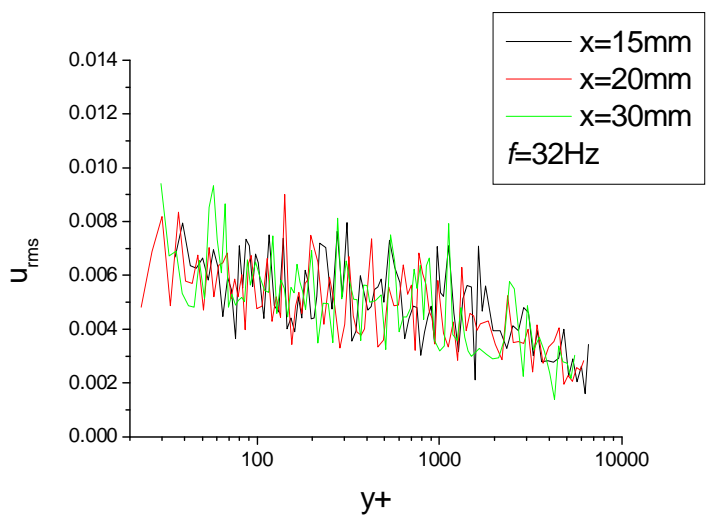

(d) 


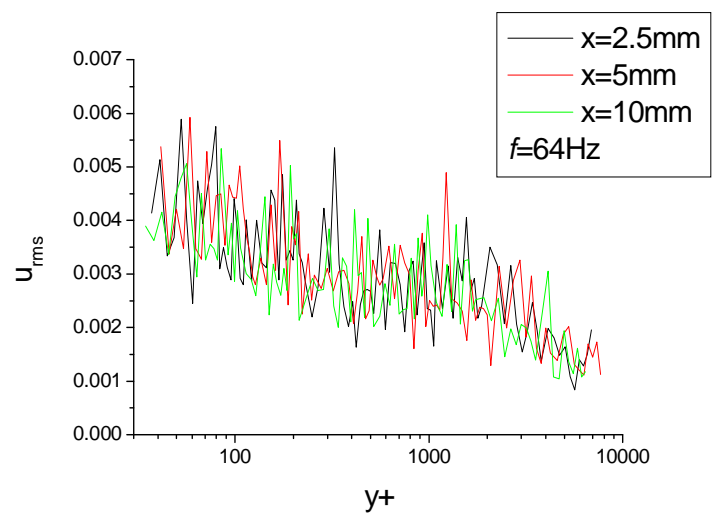

(e)

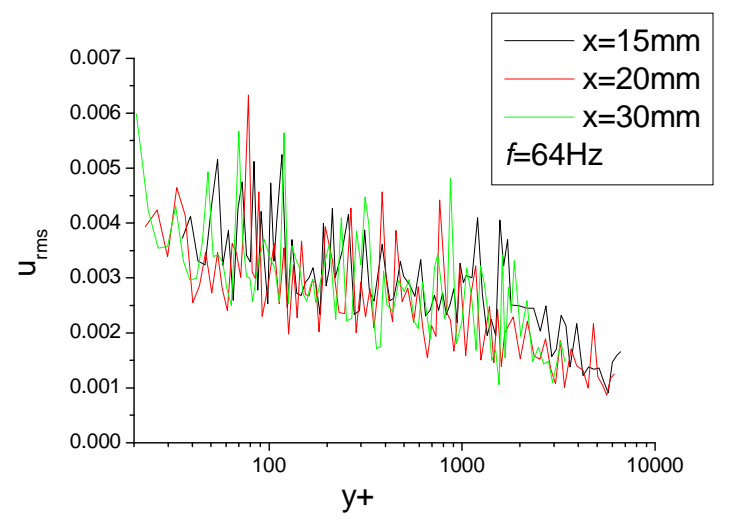

(f)

Figure 4. Decay of disturbance wave amplitude at different frequencies across turbulent boundary layer at different longitudinal stations downstream the disturbance source (a), (b) $16 \mathrm{~Hz}$; (c), (d) $32 \mathrm{~Hz}$; (e), (f) $64 \mathrm{~Hz}$.

inherent frequency components in the turbulent boundary layer is the strongest. Thereby, the $64 \mathrm{~Hz}$ perturbation in turbulent boundary layer distorts seriously and attenuations rapidly, and cannot lead to large response in the outer region $y^{+}>100$. Suppose, the frequency of the perturbation wave is $128 \mathrm{~Hz}$ (Scale 6), thus, it just fits the inherent frequency of the velocity fluctuation power spectrum in turbulent boundary layer, and its interaction with the frequency components in turbulent boundary layer is the strongest. It could attenuation most rapidly in turbulent boundary layer and cannot induce large response $y^{+}>100$.

Yao [15] studied the evolution of periodic perturbation in the log law region of turbulent boundary layer in a water channel flow by the method of inducing artificial periodic perturbation through vibrating reed. The inherent frequency in the water channel flow is below $1 \mathrm{~Hz}$, his perturbation frequency is $1-6 \mathrm{~Hz}$, which is larger than the water channel flow's inherent main frequency components. He found that the $1 \mathrm{~Hz}$ perturbation, which is approximate to the water channel flow's inherent main frequency, distorts seriously, and attenuations rapidly.

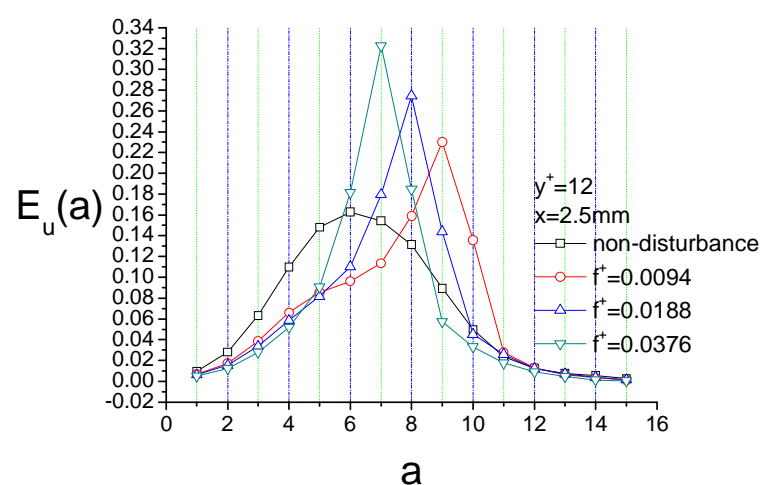

(a)

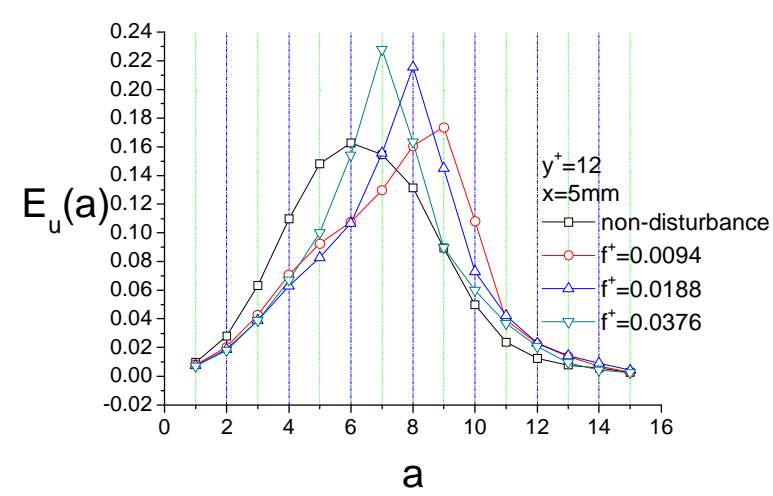

(b)

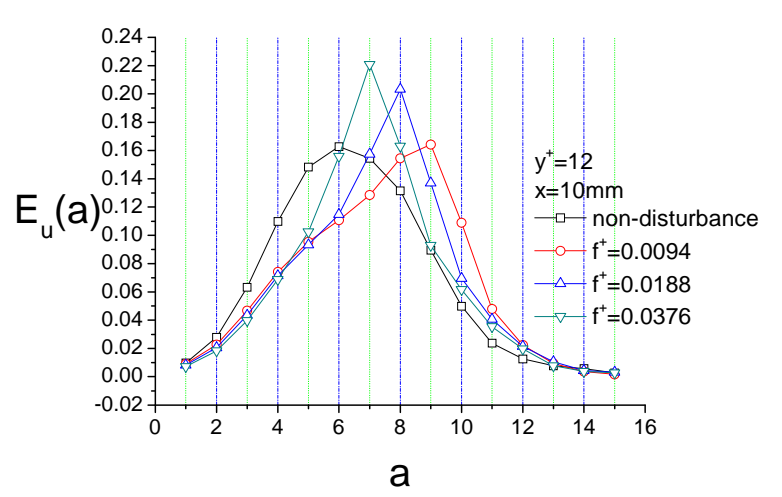

(c)

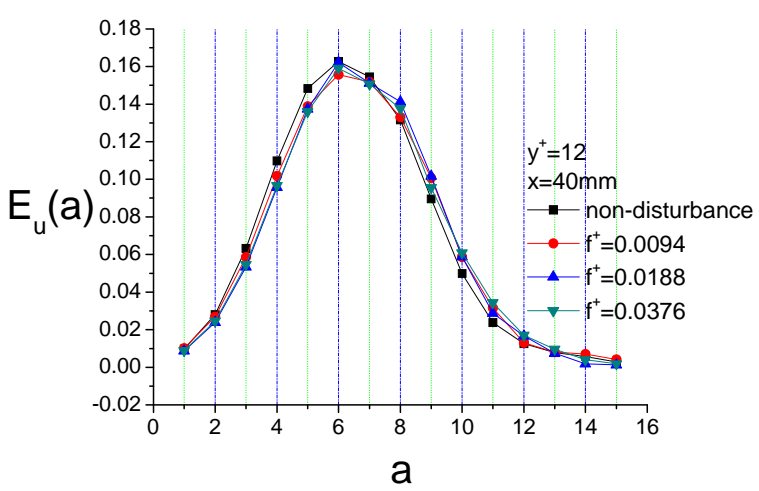

(d)

Figure 5. Single scale turbulent kinetic energy distribution across scale parameter at $y^{+}=\mathbf{1 2}$ for different longitudinal locations before and after introducing wave perturbation. 
Therefore, he concluded that the perturbation, which is most close to the inherent main frequency of the turbulent fluctuation energy in a turbulent boundary layer and has the interaction with the frequency components in the turbulent boundary layer strongest, attenuations most rapidly, and the response in the outer region of turbulent boundary layer is the weakest. As the perturbations travel to the downstream, perturbation becomes weaken gradually and its influence disappears when $x=40 \mathrm{~mm}$, the velocity fluctuation power spectrum recover to the case when no perturbation happens.

\subsection{Perturbation Wave Amplitude's Growth Rate Distribution in $x-y$ Plane}

Linear and nonlinear stability theoretical calculation indicates that there is no overall instability in turbulent boundary layer, for the turbulence is a dynamic equilibrium and stable state. Perturbation waves at different frequencies under normal circumstances trend to attenuation. Figures 6-8 are respectively the amplitude growth rate distribution of the perturbation wave of $16 \mathrm{~Hz}, 32 \mathrm{~Hz}$ and $64 \mathrm{~Hz}$. The perturbation wave amplitudes trend to attenuation on the whole, that is, the overall amplitude growth rate of the perturbation wave is $\alpha_{i}>0$, with sometimes local amplitude growth rate is $\alpha_{i}<0$, and the development of the perturbation wave amplitude is not

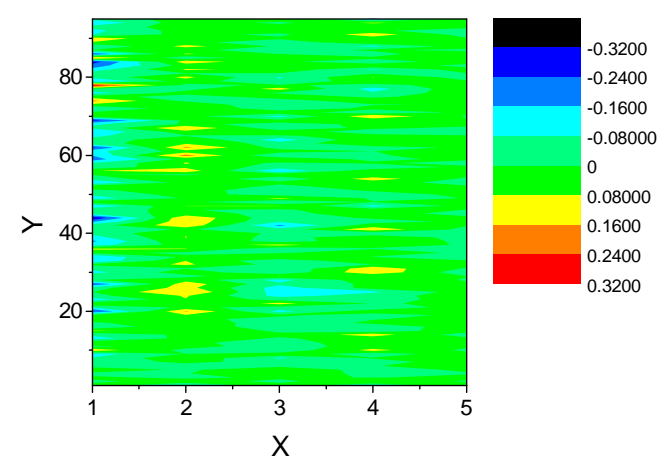

Figure 6. Amplitude attenuation rate in the $(x, y)$ plane for $16 \mathrm{~Hz}$ perturbations.

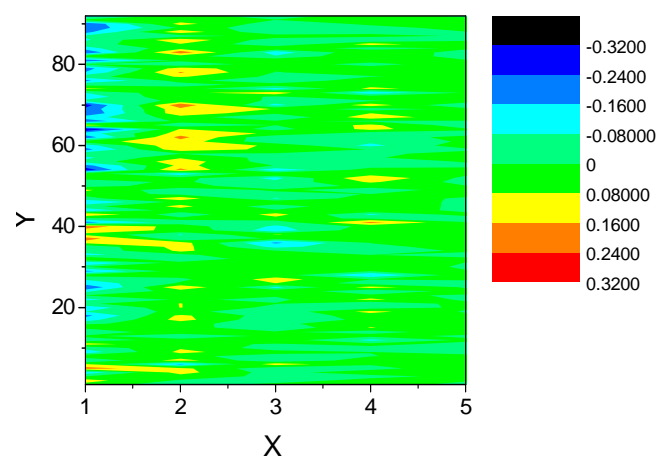

Figure 7. Amplitude attenuation rate in the $(x, y)$ plane for $32 \mathrm{~Hz}$ perturbations.

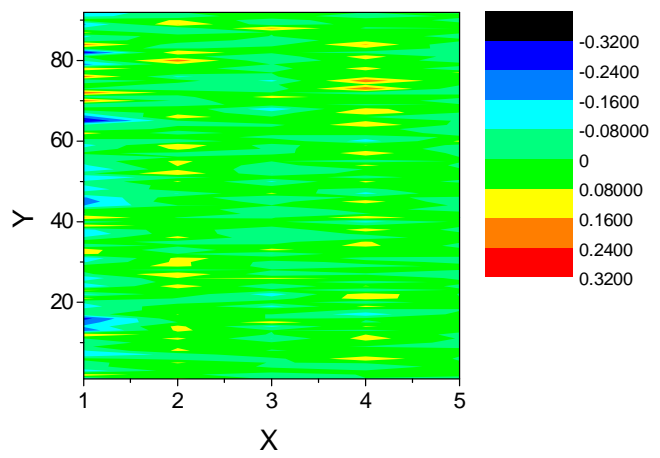

Figure 8. Amplitude attenuation rate in the $(x, y)$ plane for $64 \mathrm{~Hz}$ perturbations.

always monotonous. The attenuation rate of $16 \mathrm{~Hz}$ and $32 \mathrm{~Hz}$ perturbation in the near wall region close to the perturbation source $\left(y^{+}<100\right)$ is weak, while in the outer region is comparatively strong, but the attenuation rate along the longitudinal direction weakens gradually. The $64 \mathrm{~Hz}$ perturbation attenuations quickly in the whole flow field $\alpha_{i}>0$, because the $64 \mathrm{~Hz}$ perturbation is the most approaching to the inherent burst frequency of the turbulence and the interaction between the perturbation wave and turbulence is the strongest, perturbation wave attenuations most rapidly in turbulent boundary layer.

\section{Conclusions}

1) The inner and outer layers are not absolutely separated. They have the relationship of receptivity. The emergence of inner layer's burst is intimatly related to the outer boundary layer's flow conditions.

2) Perturbation waves at different frequencies under normal circumstances trend to attenuation. The amplitude of perturbation waves of different frequencies generally attenuations along the longitudinal direction and trends to become weaker gradually. But the perturbation wave amplitude does not always monotonously decrease with sometimes local amplitude growth rate is negative.

3) The $16 \mathrm{~Hz}$ and $32 \mathrm{~Hz}$ perturbation wave attenuations in a distance close to the perturbation source along the longitudinal direction, while their response is relatively strong in the area outside the logarithm law district. The perturbation at frequency that is the most approaching to the inherent burst frequency in turbulent boundary layer attenuations relatively rapid in the whole boundary layer.

4) The perturbation amplitude attenuation rate $\alpha_{i}$ measured in this experiment is in agreement with the results analyzed and calculated by M. Landahl's (1967) theory analysis.

\section{REFERENCES}

[1] S. J. Kline, W. C. Reynolds, F. H. Schraub and P. W. 
Runstadler, "The Structure of Turbulent Boundary Layer," Journal of Fluid Mechanics, Vol. 30, No. 4, 1967, pp. 741-774. doi: $10.1017 /$ S0022112067001740

[2] R. F. Blackweleder and R. E. Kaplan, "NATO-AGARD Conference Proceedings No. 93," Technical Editing \& Reproduction Ltd., London, 1972.

[3] G. R. Offen and S. J. Kline, "Combined Dye-Streak and Hydrogen-Bubble Visual Observations of a Turbulent Boundary Layer," Journal of Fluid Mechanics, Vol. 62, No. 2, 1974, pp. 223-239. doi:10.1017/S0022112074000656

[4] K. Rao Narasimha and M. A. Badri Narayanan, "The Bursting Phenonmena in a Turbulent Boundary Layer," Journal of Fluid Mechanics, Vol. 48, No. 2, 1971, pp. 339-396. doi:10.1017/S0022112071001605

[5] H. T. Kim, S. J. Kline and W. C. Reynolds, "The Production of Turbulence near a Smooth Wall in a Turbulent Boundary Layer," Journal of Fluid Mechanics, Vol. 50, No. 1, 1971, pp. 133-160. doi:10.1017/S0022112071002490

[6] J. Laufer and M. A. Badri Narayanan, "Mean Period of the Turbulent Production Mechanism in a Boundary Layer," Physics of Fluids, Vol. 14, No. 1, 1971, pp. 182197. doi:10.1063/1.1693271

[7] F. W. Chamber, H. D. Murphy and D. M. McEligot, "Laterally Covering Flow. Part 2, Temporal Wall Shear Stress," Journal of Fluid Mechanics, 1982, pp. 127-403.

[8] W. W. Willmarth and L. K. Sharma, "Study of Turbulent Structure with Hot Wires Smaller than the Viscous
Length," Journal of Fluid Mechanics, Vol. 142, 1984, pp. 121-149.

[9] T. S. Luchik and W. G. Tiedermann, "Time Scale and the Structure of Ejections and Bursts in Turbulent Channel Flow," Journal of Fluid Mechanics, Vol. 174, 1987, pp. 529-577. doi:10.1017/S0022112087000235

[10] W. Shu and N. Tang, "Bursting Frequency in Turbulent Boundary Layers," Acta Mechanica Sinica, Vol. 4, No. 4, 1988, pp. 291-296. doi:10.1007/BF02486661

[11] A. K. M. F. Hussain and W. C. Reynolds, "The Mechanics of an Organized Wave in Turbulent Shear Flow," Journal of Fluid Mechanics, 1970, pp. 41-241.

[12] A. K. M. F. Hussain and W. C. Reynolds, "The Mechanics of an Organized Wave in Turbulent Shear Flow. Part 2. Experimental Results," Journal of Fluid Mechanics, 1972, pp. 54-241.

[13] M. T. Landahl "A Wave-Guide Model for Turbulent Shear Flow," Journal of Fluid Mechanics, 1967, pp. 29443.

[14] V. V. Kozlov and M. P. Ramazanav, "Development of Finite-Amplitude Perturbations in Posieuille Flow," Fluid Dynamics, Vol. 18, No. 1, 1983, pp. 30-33.

[15] "The Effect of Organized Wave Perturbations on Coherent Structure in Wall Turbulence," Acta Mechanica Sinica, Vol. 23, No. 4, 1991, pp. 47-54 (in Chinese).

[16] W. W. Willmarth and C. E. Wooldridge, "Measurements of the Fluctuating Pressure at the Wall beneath a Thick Turbulent Boundary Layer," Journal of Fluid Mechanics, Vol. 14, 1962, pp. 14-187. 